\title{
Positive solutions for one-dimensional p-Laplacian boundary value problems with nonlinear parameter
}

\author{
Ahmed Omer Mohammed Abubaker \\ Department of Mathematics, Faculty of Education, University of Khartoum, Omdurman, 406, Sudan \\ E-mail: wadomar877@hotmail.com
}

Copyright (C)2014 Ahmed Abubaker. This is an open access article distributed under the Creative Commons Attribution License, which permits unrestricted use, distribution, and reproduction in any medium, provided the original work is properly cited.

\section{Abstract}

In this paper, we establish existence of positive solutions of the nonlinear problems of one - dimensional p-Laplacian with nonlinear parameter

$$
\varphi_{p}\left(u^{\prime}(t)\right)^{\prime}+a(t) f(\lambda, u)=0, \quad t \in(0,1), \quad u(0)=u(1)=0
$$

where $a: \Omega \rightarrow R$ is continuous and may change sign, $\lambda>0$ is a parameter, $f(\lambda, 0)>0$ for all $\lambda>0$. By applying Leray-Schauder fixed point theorem we obtain the existence of positive solutions.

Keywords: p-Laplacian, Positive solutions, Leray-Schauder fixed point theorem, nonlinear parameter.

\section{Introduction}

The boundary value problem for one- dimensional p-Laplacian

$\left\{\begin{array}{l}\varphi_{p}\left(u^{\prime}(t)\right)^{\prime}+\lambda a(t) f(u)=0, \quad t \in(0,1), \\ u(0)=u(1)=0\end{array}\right.$

where $\varphi_{p}(u(t))=|u|^{p-2} \quad u, \quad p>1$, has been studied extensively. For details, see for example, Refs $[1,2,5]$, in the case $\mathrm{p}=2$ see [6], and for case $\lambda=1$, see $[7,8,9]$.

In a recent paper [4], Hai considered the boundary value problem

$\begin{cases}\Delta u+\lambda a(t) f(u)=0, & t \in \Omega, \\ u=0, & t \in \partial \Omega,\end{cases}$

where $\Omega$ is a bounded domain in $R^{N}, a: \Omega \rightarrow R$ is continuous and changes its sign, $f(0)>0$, and $\lambda>0$ is sufficiently small, under the following assumptions

(A1) $f:[0, \infty) \rightarrow R$ is continuous and $f(0)>0$.

(A2) $a: \bar{\Omega} \rightarrow R$ is continuous, $a \not \equiv 0$, and there exists a number $k>1$ such that 
$\int_{\Omega} G(t, s) a^{+}(s) d s \geq k \int_{\Omega} G(t, s) a^{-}(s) d s, \quad t \in \Omega$,

where $a^{+}$(resp. $a^{-}$) is the positive (resp. negative) part of a, and $G(t, s)$ is the Green's function of $-\Delta$ with Dirichlet boundary conditions.

They obtained the following interesting result:

Theorem A. Let (A1), (A2) hold. Then there exists a positive number $\lambda^{\star}$, such that (2) has a positive solution for $\lambda<\lambda^{\star}$. In another recent paper [3], Ma et al investigated the boundary value problem

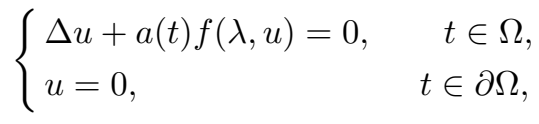

By applying Leray-Schauder fixed point theorem they obtained that the problem (3) has a positive solution for $\lambda<\lambda^{\star}$. Motivated by the results mentioned in [3,4] above, in this paper we study the existence of positive solutions of the nonlinear one- dimensional p-Laplacian

$\left\{\begin{array}{l}\varphi_{p}\left(u^{\prime}(t)\right)^{\prime}+a(t) f(\lambda, u)=0, \quad t \in(0,1), \\ u(0)=u(1)=0\end{array}\right.$

where $\varphi_{p}(u(t))=|u|^{p} \quad u, p>1$, and hence $\varphi_{p}\left(u^{\prime}(t)\right)^{\prime}$ is the one- dimensional p-Laplacian, and $a: \Omega \rightarrow R$ is continuous and changes its sign, $\lambda>0$ is a parameter, $f(\lambda, 0)>0$ for all $\lambda>0$.

The following hypotheses are adopted throughout this paper:

(H1) $f \in C([0, \infty) \times[0, \infty), R)$ and $f(\lambda, 0)>0$ for all $\lambda>0, f(0, u)=0$ for $u \in[0, \infty)$

(H2) $a: \bar{\Omega} \rightarrow R$ is continuous, $a \not \equiv 0$, and there exists a number $k>1$ such that

$\int_{0}^{t} \varphi_{p}^{-1}\left(h\left(a^{+}\right)+\int_{0}^{s} a^{+}(\tau) d \tau\right) d s \geq k \int_{0}^{t} \varphi_{p}^{-1}\left(h\left(a^{-}\right)+\int_{0}^{s} a^{-}(\tau) d \tau\right) d s$,

where $h: L^{1}(0,1) \rightarrow R$ is continuous function satisfying

$\int_{0}^{1} \varphi_{p}^{-1}\left(h(a)+\int_{0}^{s} a(\tau) d \tau\right) d s=0$.

The main result of this paper is as follows

Theorem 1.1. Let (H1), (H2) hold. Then there exists a positive number $\lambda^{\star}$, such that (4) has a positive solution for $\lambda<\lambda^{\star}$.

The proof of theorem 1.1 is based on the Leray -Schauder theorem see [10], for more details.

Remark 1.1. If we let $f(\lambda, u):=\lambda f(u)$ and $\varphi_{p}(u)=\Delta u$, in (4), then (4) reduces to (2), (H1) reduces to (A1). Therefore, [4, Theorem 1.1], (see Theorem A above), is the direct consequence of Theorem 1.1.

Clearly, Theorem 1.1 is an extension and improvement of the existence results in [4, Hai], [3, Ma]

The rest of this paper is arranged as follows. In Section 2, we will give some notations and preliminary results, in Section 3, we prove Theorem 1.1 via the Leray - Schauder fixed point theorem.

\section{Preliminaries}

Throughout the paper, we assume that $f(\lambda, u)=f(\lambda, 0)$ for $u \leq 0$ and given $\lambda>0$.

For $u \in C_{0}^{1}[0,1]$, define the operator $T$ by

$T u(t)=\int_{0}^{t} \varphi_{p}^{-1}\left(h\left(a^{+}\right) f(\lambda, u(s))+\int_{0}^{s} a^{+}(\tau) f(\lambda, u(s)) d \tau\right) d s$.

It's not difficult to see that $T: C_{0}^{1}[0,1] \rightarrow C_{0}^{1}[0,1]$ is completely continuous.

Lemma 2.1. Let $0<\delta<1$. Then there exists a positive number $\bar{\lambda}$ such that, for $0<\lambda<\bar{\lambda}$, the equation

$\varphi_{p}\left(u^{\prime}(t)\right)^{\prime}=-a^{+}(t) f(\lambda, u), \quad 0<t<1 \quad, \quad u(0)=u(1)=0$

has a positive solution $\tilde{u}_{\lambda}$ with $\left\|\tilde{u}_{\lambda}\right\|_{0} \rightarrow 0$ as $\lambda \rightarrow 0$, and 
$\tilde{u}_{\lambda}(t) \geq \delta f(\lambda, 0) p(t), \quad t \in \Omega$,

where $p(t)=\int_{0}^{t} \varphi_{p}^{-1}\left(h\left(a^{+}\right)+\int_{0}^{s} a^{+}(\tau) d \tau\right) d s$.

Proof. We shall apply the Leray-Schauder fixed point theorem to prove that $T$ has a fixed point for $\lambda$ small. Let $\varepsilon>0$ be such that

$f(\lambda, u) \geq \delta f(\lambda, 0), \quad$ for $0 \leq u \leq \varepsilon$.

From $f(0, u) \equiv 0, \forall u \geq 0$, we can suppose that $0<\lambda<\varepsilon \lambda / 2\|p\|_{0} \tilde{f}(\lambda, \varepsilon)$, for given $\lambda>0$, where $\tilde{f}(\lambda, t)=$ $\max _{0 \leq s \leq t} f(\lambda, s)$. Then there exists $A_{\lambda} \in(0, \varepsilon)$ such that

$\frac{\tilde{f}\left(\lambda, A_{\lambda}\right)}{\lambda A_{\lambda}}=\frac{1}{2 \lambda\|p\|_{0}}$.

Let $u \in C_{0}^{1}[0,1]$ and $\theta \in(0,1)$ be such that $u=\theta T u$. Then we have

$\|u\|_{0} \leq \lambda\|p\|_{0} \frac{\tilde{f}\left(\lambda,\|u\|_{0}\right)}{\lambda}$

or

$\frac{\tilde{f}\left(\lambda,\|u\|_{0}\right)}{\lambda\|u\|_{0}} \geq \frac{1}{\lambda\|p\|_{0}}$

which implies that $\|u\|_{0} \neq A_{\lambda}$. Note that $A_{\lambda} \rightarrow 0$ as $\lambda \rightarrow 0$. By the Leray-Schauder fixed point theorem, $T$ has a fixed point $\tilde{u}_{\lambda}$ with $\left\|\tilde{u}_{\lambda}\right\|_{0} \leq A_{\lambda}<\varepsilon$. Consequently, $\tilde{u}_{\lambda}(t) \geq \lambda \delta \frac{f(\lambda, 0)}{\lambda} p(t), t \in[0,1]$, and the proof is completed.

\section{Proof of the Theorem 1.1}

Let $q(t)=\int_{0}^{t} \varphi_{p}^{-1}\left(h\left(a^{-}\right)+\int_{0}^{s} a^{-}(\tau) d \tau\right) d s$. By (H2), there exist positive numbers $\alpha, \gamma \in(0,1)$ such that

$q(t)|f(\lambda, s)| \leq \gamma p(t) f(\lambda, 0)$

for $s \in[0, \alpha], t \in[0,1]$. Fix $\delta \in(\gamma, 1)$ and let $\lambda^{*}>0$ be such that

$\left\|\tilde{u}_{\lambda}\right\|_{0}+\lambda \delta \frac{f(\lambda, 0)}{\lambda}\|p\|_{0} \leq \alpha$

for $0<\lambda<\lambda^{*}$, where $\tilde{u}_{\lambda}$ is given by Lemma 2.1 , and

$|f(\lambda, x)-f(\lambda, y)| \leq f(\lambda, 0)\left(\frac{\delta-\gamma}{2}\right)$

for $x, y \in[-\alpha, \alpha]$ with $|x-y| \leq \lambda^{*} \delta \frac{f(\lambda, 0)}{\lambda}\|p\|_{0}$.

Let $0<\lambda<\lambda^{*}$. We look for a solution $u_{\lambda}$ of (4) of the form $\tilde{u}_{\lambda}+v_{\lambda}$. Thus $v_{\lambda}$ satisfies

$$
\begin{aligned}
v_{\lambda}(t) & =\int_{0}^{t} \varphi_{p}^{-1}\left(h\left(a f\left(\lambda, \tilde{u}_{\lambda}+v_{\lambda}\right)\right)+\int_{0}^{s} a(\tau) f\left(\lambda, \tilde{u}_{\lambda}+v_{\lambda}\right) d \tau\right) d s \\
& -\int_{0}^{t} \varphi_{p}^{-1}\left(h\left(a^{+} f\left(\lambda, \tilde{u}_{\lambda}\right)\right)+\int_{0}^{s} a^{+}(\tau) f\left(\lambda, \tilde{u}_{\lambda}\right) d \tau\right) d s \quad, \quad 0<t<1 .
\end{aligned}
$$

For each $w \in C_{0}^{1}[0,1]$, let $v=T w$ be the solution of $\begin{aligned} v(t) & =\int_{0}^{t} \varphi_{p}^{-1}\left(h\left(a f\left(\lambda, \tilde{u}_{\lambda}+\omega\right)\right)+\int_{0}^{s} a(\tau) f\left(\lambda, \tilde{u}_{\lambda}+\omega\right) d \tau\right) d s \\ & -\int_{0}^{t} \varphi_{p}^{-1}\left(h\left(a^{+} f\left(\lambda, \tilde{u}_{\lambda}\right)\right)+\int_{0}^{s} a^{+}(\tau) f\left(\lambda, \tilde{u}_{\lambda}\right) d \tau\right) d s \quad, \quad 0<t<1 .\end{aligned}$ 
Then $T: C_{0}^{1}[0,1] \rightarrow C_{0}^{1}[0,1]$ is completely continuous. Let $v \in C_{0}^{1}[0,1]$ and $\theta \in(0,1)$ be such that $v=\theta T v$. Then we have

$$
\begin{gathered}
v=\theta \int_{0}^{t} \varphi_{p}^{-1}\left(h\left(a f\left(\lambda, \tilde{u}_{\lambda}+v\right)\right)+\int_{0}^{s} a(\tau) f\left(\lambda, \tilde{u}_{\lambda}+v\right) d \tau\right) d s \\
-\theta \int_{0}^{t} \varphi_{p}^{-1}\left(h\left(a^{+} f\left(\lambda, \tilde{u}_{\lambda}\right)\right)+\int_{0}^{s} a^{+}(\tau) f\left(\lambda, \tilde{u}_{\lambda}\right) d \tau\right) d s .
\end{gathered}
$$

We claim that $\|v\|_{0} \neq \delta f(\lambda, 0)\|p\|_{0}$. Suppose on the contrary that $\|v\|_{0}=\delta f(\lambda, 0)\|p\|_{0}$. Then, by (8) and (9), we obtain and

$$
\left\|\tilde{u}_{\lambda}+v\right\|_{0} \leq\left\|\tilde{u}_{\lambda}\right\|_{0}+\|v\|_{0} \leq \alpha
$$

$$
\left|f\left(\lambda, \tilde{u}_{\lambda}+v\right)-f\left(\lambda, \tilde{u}_{\lambda}\right)\right| \leq f(\lambda, 0) \frac{\delta-\gamma}{2},
$$

which together with $(7)$ implies that

$$
\begin{aligned}
|v(t)| & \leq \lambda \frac{\delta-\gamma}{2} \frac{f(\lambda, 0)}{\lambda} p(t)+\lambda \gamma \frac{f(\lambda, 0)}{\lambda} p(t) \\
& =\lambda \frac{\delta+\gamma}{2} \frac{f(\lambda, 0)}{\lambda} p(t),
\end{aligned}
$$

In particular

$$
\|v\|_{0} \leq \lambda \frac{\delta+\gamma}{2} \frac{f(\lambda, 0)}{\lambda}\|p\|_{0}<\lambda \delta \frac{f(\lambda, 0)}{\lambda}\|p\|_{0}
$$

a contradiction, and the claim is proved. By the Leray -Schauder fixed point theorem, $T$ has a fixed point $v_{\lambda}$ with $\left\|v_{\lambda}\right\|_{0} \leq \delta f(\lambda, 0)\|p\|_{0}$. Hence $v_{\lambda}$ satisfies (10) and, using Lemma 2.1, we obtain

$$
\begin{aligned}
u_{\lambda}(t) & \geq \tilde{u}_{\lambda}(t)-v_{\lambda}(t) \\
& \geq \lambda \delta \frac{f(\lambda, 0)}{\lambda} p(t)-\lambda \frac{\delta+\gamma}{2} \frac{f(\lambda, 0)}{\lambda} p(t) \\
& =\lambda \frac{\delta-\gamma}{2} \frac{f(\lambda, 0)}{\lambda} p(t),
\end{aligned}
$$

i.e., $u_{\lambda}$ is a positive solution of (4). This completes the proof of Theorem 1.1.

\section{Acknowledgements}

First i would like to thank the Editor of the International Journal of Applied Mathematical Research for his good advice, and now i have the pleasure and joy to take this opportunity to thank all people who have supported and accompanied with me. Firstly I would like to thank my advisor Prof. Ma Ruyun for the continuous support of me, I give my sincere thanks to the Ibrahim Omar Osman, my thanks extend to University of Khartoum.

\section{References}

[1] Yong-Hoon Lee, Inbo Sim; Global bifurcation phenomena for singular one-dimensional p-Laplacian; J. Differential Equations 229 (2006) 229 - 256.

[2] Justino Sanchez; Multiple positive solutions of singular eigenvalue type problems involving the one-dimensional pLaplacian; J. Math. Anal. Appl. 292 (2004) 401 - 414.

[3] Ruyun Ma, et al;Existence of positive solutions to a class of elliptic boundary value problems with nonlinear parameter; to appear.

[4] D. D. Hai; Positive solutions to a class of elliptic boundary value problems; J. Math. Anal. Appl., 227 (1998), 195 - 199.

[5] R.P. Agarwal, H. L, D. ORegan; Eigenvalues and the one-dimensional p-Laplacian; J. Math. Anal. Appl. 266 (2002) $383-400$. 
[6] B. Im, E. Lee, Y.H. Lee; A global bifurcation phenomena for second order singular boundary value problems; J. Math. Anal. Appl. 308 (2005) $61-78$.

[7] L. Kong, J. Wang; Multiple positive solutions for the one-dimensional p-Laplacian; Nonlinear Anal. 42 (2000) 1327 1333.

[8] L. Kong, Wenjie Gso; A singular boundary value problem for the one-dimensional p-Laplacian; J. Math. Anal. Appl 201 (1996) $851-866$.

[9] J. Wang; The existence of positive solutions for the one-dimensional p-Laplacian; Proc. Amer. Math. Soc. 125 (1997) $2275-2283$.

[10] Jean Mawhin; Lerary - Schauder degree: A half century of extensions and applications; Topological Methods in Nonlinear Analysis, 14, 1999, $195-228$. 